\title{
Efecto de la Temperatura, el pH y el Contenido en Sólidos sobre los Compuestos Fenólicos y la Actividad Antioxidante del Extracto de Bixa orellana L.
}

\author{
Cindy T. Sepúlveda y José E. Zapata* \\ Facultad de Ciencias Farmacéuticas y Alimentarias, Dpto. de Alimentos, Universidad de Antioquia, Calle 70 \# 52-21, \\ Apartado aéreo AA1226, Medellín, Colombia. (e-mail: tatiana1827@gmail.com; edgar.zapata@udea.edu.co)
}

* Autor a quien debe ser dirigida la correspondencia

Recibido Oct. 16, 2018; Aceptado Dic. 19, 2018; Versión final Feb. 8, 2019, Publicado Oct. 2019

\begin{abstract}
Resumen
El objetivo del presente trabajo fue evaluar el efecto de la temperatura, el pH y el contenido en sólidos solubles, sobre la velocidad de reacción, en la degradación térmica de los fenoles totales del extracto etanólico de semillas de Bixa orellana L. Se determina también la estabilidad de fenoles totales y su actividad antioxidante durante 91 días a condiciones de almacenamiento de $-20,8,23$ y $37^{\circ} \mathrm{C}$. Los resultados mostraron que la degradación de fenoles totales, se ve afectada por la temperatura y los sólidos solubles, siguiendo una cinética de primer orden, tanto en condiciones de procesamiento como de almacenamiento. Por su parte, la actividad antioxidante (ABTS y FRAP), se ve afectada por la temperatura de almacenamiento. Los tiempos de vida media estuvieron entre 27.74-38.25; 27.99-36.51 y 22.18-35.98 días ${ }^{-1}$, para compuestos fenólicos, ABTS y FRAP, respectivamente. Mientras que la energía de activación fue 4.08, 3.24 y $5.52 \mathrm{KJ} \mathrm{mol}^{-1}$ para fenoles totales, ABTS y FRAP, respectivamente. Se concluye que la actividad antioxidante de estos extractos depende de su contenido de compuestos fenólicos los cuales son afectados por la temperatura y por el contenido de sólidos solubles.
\end{abstract}

Palabras clave: antioxidante; actividad biológica; cinética de primer orden; fenoles totales; estabilidad térmica; energía de activación

\section{Effect of Temperature, $\mathrm{pH}$ and Solids Content on Phenolic Compounds and Antioxidant Activity of Bixa orellana L. Extract}

\begin{abstract}
The objective of this work was to evaluate the effect of temperature, $\mathrm{pH}$ and soluble solids on reaction rate in phenolic compound degradation of Bixa orellana L. Also, the stability of phenolic compounds and their antioxidant activity during 91 days under storage condition of $-20,8,23$ and $37{ }^{\circ} \mathrm{C}$ were determined. The results showed that phenolic compound degradation depends on temperature and soluble solids, it followed a first-order kinetic for both, process and storage conditions. In addition, its antioxidant activities (ABTS and FRAP) were affected by storage temperature. The half-life times were between $27.74-38.25 ; 27.99-36.51$ and 22.18-35.98 day $^{-1}$, for phenolic compound, ABTS and FRAP, respectively. The activation energy was 4.08, 3.24 and $5.52 \mathrm{KJ} \mathrm{mol}^{-1}$, for the phenolic compounds, ABTS and FRAP, respectively. It was concluded that the antioxidant activity of these extracts depends on phenolic compounds content which is affected by temperature and by soluble solids.
\end{abstract}




\section{INTRODUCCIÓN}

El achiote (Bixa orellana L.) es una especie ampliamente conocida por la utilidad de sus semillas como colorante en la industria cosmética y alimentaria (Campos et al., 2011). Ha tenido una gran aplicación en la medicina tradicional de muchos países (Bachir et al., 2014) despertando interés en la identificación y el estudio de su composición. Los compuestos fenólicos que constituyen uno de los metabolitos secundarios presentes en las plantas (Moo-Huchin et al., 2015), los cuales son de considerable importancia fisiológica y morfológica ya que intervienen en los procesos de crecimiento y reproducción en las plantas, proporcionando protección contra microorganismos (Silva et al., 2018). Los compuestos fenólicos, exhiben una amplia gama de propiedades biológicas, en las que se incluyen las propiedades antimicrobianas, (Koolen et al., 2013; Do Prado et al., 2014) antiinflamatoria (Cartarino et al., 2016), antialérgica (Agati et al., 2012), antiviral (Zhang et al., 2014), anticancerígena (Gad et al,.2016), antioxidante (Zhang et al., 2014), entre otras (Lima et al.. 2018; Araujo et al,. 2018). Sin embargo dichos compuestos, pueden ser vulnerables a la luz, temperatura (Kırca et al., 2007; Albarici y Pessoa, 2012), contenido de sólidos (Kırca et al., 2007), pH (Reyes y Cisneros-Zevallos, 2007; He et al., 2015), oxígeno (Kırca et al., 2007; Garzón, 2008), ácido ascórbico (Garzón, 2008) y tipo de solvente en que se tengan los extractos, en cuyo caso, en el agua se tiene una de las menores estabilidades térmica (Padmashree et al., 2014).

Se han llevado a cabo estudios orientados a establecer la estabilidad térmica de compuestos fenólicos como es el caso de antocianinas en zanahoria negra (Kırca et al., 2007), en hojas de Solanum nigrum $L$. (Padmashree et al., 2014); papas, zanahorias y uvas (Reyes y Cisneros-Zevallos, 2007; He et al., 2015). Sin embargo, el efecto que ejerce la temperatura sobre los compuestos fenólicos depende de la matriz de la cual se hayan extraído dichos compuestos (Padmashree et al., 2014) y tampoco se encuentran estudios que evalúen el efecto de la temperatura o el $\mathrm{pH}$, sobre la estabilidad de la actividad antioxidante de dichos compuestos. El objetivo del presente trabajo fue evaluar el efecto que tiene la temperatura ( $T$ ), los sólidos solubles (SS) y el pH, sobre la velocidad de degradación de los compuestos fenólicos extraídos de semillas de $B$. orellana L. y sobre velocidad de pérdida de actividad antioxidante, tanto en temperaturas típicas de procesos de alimentos $\left(70,80\right.$ y $\left.90^{\circ} \mathrm{C}\right)$, como en temperaturas de almacenamiento $\left(-20,8,23\right.$ y $\left.37^{\circ} \mathrm{C}\right)$.

\section{MATERIALES Y MÉTODOS}

La metodología presenta la caracterización de $B$. orellana, preparación de las muestras y los diferentes métodos analíticos que se le realizaron a la muestra además de los métodos para la determinación de la cinética de degradación térmica de fenoles totales y la pérdida de actividad antioxidante a condiciones de almacenamiento.

\section{Material vegetal}

Las semillas de $B$. orellana L. se recolectaron en un predio particular del municipio de San Luis (150 m.s.n.m, $28 \stackrel{\circ}{ }$ ) $)$, departamento de Antioquia, Colombia. Un ejemplar reposa en el Herbario de la Universidad de Antioquia (№ HUA 108450), identificado como Bixa orellana L. variedad roja.

\section{Preparación de los extractos}

Las semillas fueron secadas en estufa convencional (Thermo Scientific Heratherm) a temperatura de $37 \pm 0.2$ ${ }^{\circ} \mathrm{C}$. El material vegetal seco fue sometido a un proceso de extracción sólido-líquido a $4 \pm 0.2^{\circ} \mathrm{C}$ con etanol al $95 \%$.

\section{Determinación del contenido de fenoles totales (FT)}

El contenido de fenoles totales en el extracto se determinó por el método de Folin-Ciocalteu (Singleton y Rossi, 1965), donde a $500 \mu \mathrm{L}$ del extracto se le adicionaron $250 \mu \mathrm{L}$ del reactivo Folin-Ciocalteu, posteriormente se adicionó $1250 \mu \mathrm{L}$ de $\mathrm{Na}_{2} \mathrm{CO}_{3}$ al $20 \%$. La mezcla se dejó en reposo durante $2 \mathrm{~h}$ en la oscuridad. Finalmente, se midió la absorbancia a $725 \mathrm{~nm}$ en un espectrofotómetro (UV-1700, Shimadzu Europe) y los resultados fueron calculados mediante extrapolación en una curva de calibración con ácido tánico como patrón entre 0.01 y $0.1 \mathrm{mg} \cdot \mathrm{mL}^{-1}$. Las determinaciones se realizaron por triplicado y se expresaron como equivalentes de ácido tánico por gramos de $\left(\mathrm{mgAT}_{\mathrm{g}} \mathrm{g}^{-1}\right)$.

\section{Reacción con el radical 2,2’azino-bis (3-etilbenzotiazolin-6- ácido sulfónico) (ABTS*)}

Se determinó de acuerdo a la metodología descrita por Re et al. (1999) con algunas modificaciones. Se preparó una solución de ABTS de $7 \mathrm{mM}$ y una solución de persulfato potásico de $2.45 \mathrm{mM}$. La mezcla y se incubó por $16 \mathrm{~h}$ a temperatura ambiente $\left( \pm 25^{\circ} \mathrm{C}\right)$ para formar el radical ABTS $\bullet+.1 \mathrm{~mL}$ del radical se mezcló 
con $100 \mu \mathrm{L}$ de extracto y se llevó a incubación a $30^{\circ} \mathrm{C}$ durante 30 minutos en la oscuridad. Por último fue medida la absorbancia a una longitud de onda de $730 \mathrm{~nm}$ en un espectrofotómetro (UV-1700, Shimadzu Europe). Las determinación se realizó por triplicado y los resultados fueron calculados mediante extrapolación en una curva de calibración entre 50 y $400 \mu \mathrm{M}$ con Trolox como patrón. Los valores obtenidos se expresaron como micromoles equivalentes de Trolox por gramo de extracto ( $\left.\mu \mathrm{molET} . \mathrm{g}^{-1}\right)$.

\section{Medida de la capacidad reductora del Fe+3 (FRAP)}

Se llevó a cabo mediante la metodología propuesta por Pulido et al. (2000), en la cual el reactivo de FRAP (2,4,6-Tripiridil-s-Triazina, cloruro de hierro y buffer de acetato de sodio) recién preparado y calentado a una temperatura de $37^{\circ} \mathrm{C}$, se mezcló con agua destilada y extracto o estándar y se incubó a $37^{\circ} \mathrm{C}$ por 30 minutos. El análisis se realizó por triplicado y se leyó la absorbancia a $595 \mathrm{~nm}$ en un espectrofotómetro (UV-1700, Shimadzu Europe). Los resultados fueron calculados mediante extrapolación en una curva de calibración entre 50 y $400 \mu \mathrm{M}$ con Trolox como patrón y se expresaron como micromoles equivalentes de Trolox por gramo de extracto $\left(\mu \mathrm{molET} . \mathrm{g}^{-1}\right)$.

\section{Efecto de la temperatura, el pH y los sólidos solubles sobre la velocidad de degradación de FT}

Por ser las variables más relevantes en el procesamiento de alimentos, se escogió evaluar el efecto de temperatura, $\mathrm{pH}$ y SS sobre la velocidad de degradación de FT en el extracto de B. orellana L., para ello se aplicó un diseño factorial (DOE) donde se consideraron los factores: $\mathrm{pH}$ del medio en el que se encuentra el extracto con valores de 5.5 y 8 , temperatura con valores de $70{ }^{\circ} \mathrm{C}, 80^{\circ} \mathrm{C}$ y $90^{\circ} \mathrm{C}$ usando un baño en seco (Labnet AccuBlock), se ajustó el contenido de sólidos solubles a 8, 14 y $20^{\circ}$ Brix usando un refractómetro (AR200 Reichert), los tubos donde se realizó el experimento permanecieron cerrados para evitar la evaporación por el calentamiento y se determinó el contenido de fenoles totales cada 90 minutos durante 9 horas para calcular la constante de velocidad de reacción k (Ecuación 1) como variable respuesta del DOE; además se calculó: la energía de activación Ea (Ecuación 2), el coeficiente de temperatura (Q10) (Ecuación 3) y el t1/2 (Ecuación 4).

$$
\begin{aligned}
& \operatorname{Ln} C=\operatorname{LnC}_{0}-k_{1} t \\
& \operatorname{Ln} k=\operatorname{Ln} k_{0}-\frac{E_{a}}{R T} \\
& Q_{10}=\left(k_{2} / k_{1}\right)^{\frac{10}{T_{2}-T_{1}}} \\
& -\operatorname{Ln}\left(\frac{0.5}{1}\right)=k_{1} t_{\frac{1}{2}}
\end{aligned}
$$

\section{Cinética de degradación de FT y pérdida de actividad antioxidante a condiciones de almacenamiento}

La degradación de los compuestos fenólicos y la pérdida de actividad antioxidante del extracto etanólico de B. orellana L. fue estudiado a pH 6.67 y $12.69{ }^{\circ}$ Brix almacenado a $-20{ }^{\circ} \mathrm{C}, 8{ }^{\circ} \mathrm{C}, 23^{\circ} \mathrm{C}$ y $37^{\circ} \mathrm{C}$. Las determinaciones se realizaron cada 7 días durante 3 meses. Además, se calcularon los siguientes parámetros cinéticos: k (Ecuación 1), Ea (Ecuación 2), el Q10 (Ecuación 3), y el t1/2 (Ecuación 4).

\section{Análisis estadísticos}

Los datos fueron procesados estadísticamente con el programa STAT-EASE de Design-Expert, versión 7.1.6 (Stat-Ease, EE.UU). Para los datos con distribución normal y homogeneidad de varianzas, se aplicó el análisis de la varianza (ANOVA) (Montgomery, 2005), con un nivel de confianza del 95\%, el cual incluyó la significancia estadística de cada uno de los términos del modelo ajustado (Valor - P), los coeficientes estimados en cada término $\left(\beta_{i}\right)$ y el coeficiente de determinación del modelo $\left(\mathrm{R}^{2}\right)$.

\section{RESULTADOS Y DISCUSIÓN}

A continuación se presentan los resultados obtenidos del presente trabajo, empezando por el efecto de los factores sobre las respuestas, a temperaturas de proceso, seguidos por los resultados a condiciones de almacenamiento. 


\section{Efecto de la temperatura, el pH y los sólidos solubles sobre la velocidad de degradación de FT}

La degradación térmica de FT siguió un modelo de reacción de primer orden, que coincide con los resultados encontrados por otros estudios relacionados con los compuestos fenólicos provenientes de otros sustratos vegetales (Kırca et al., 2007; Fischer et al., 2013; De Paepe et al., 2014; Henríquez et al., 2014). En la Tabla 1 se muestran las corridas experimentales del DOE, con los factores T, SS y pH y su respuesta $\mathrm{k}$, que fue calculada a partir de la ecuación 1 para cada corrida. Valores de igual magnitud fueron obtenidos por Reyes y Cisneros-Zevallos (2007), trabajando con antocianinas de papa roja, papa morada, zanahorias y uvas, así como los de Kırca et al. (2007), quienes estudiaron las antocianinas de zanahoria negra a pH ácidos.

Tabla 1: Diseño factorial y parámetros cinéticos de la degradación de los fenoles totales del extracto de semillas de Bixa orelllana. Los números entre paréntesis corresponden a los coeficientes de determinación.

\begin{tabular}{|c|c|c|c|c|c|c|c|c|}
\hline \multirow{2}{*}{ Corrida } & \multirow{2}{*}{$\begin{array}{c}\text { Temperatura } \\
\left.{ }^{\circ} \mathrm{C}\right)\end{array}$} & \multirow{2}{*}{$p H$} & $S S$ & $k$ & $t_{1 / 2}$ & $E a$ & \multicolumn{2}{|c|}{$Q_{10}$} \\
\hline & & & ( ${ }^{\circ}$ Brix $)$ & $\left(\mathrm{min}^{-1}\right)$ & (min) & $\left(\mathrm{KJ} \mathrm{mol}^{-1}\right)$ & $(70 / 80)$ & $(80 / 90)$ \\
\hline 14 & 70 & 5.5 & 8 & $8.0 \times 10^{-4}(0.92)$ & 818 & \multirow{3}{*}{$90(0.87)$} & \multirow{3}{*}{4.1} & \multirow{3}{*}{1.4} \\
\hline 3 & 80 & 5.5 & 8 & $3.5 \times 10^{-3}(0.95)$ & 200 & & & \\
\hline 11 & 90 & 5.5 & 8 & $4.8 \times 10^{-3}(0.84)$ & 144 & & & \\
\hline 15 & 70 & 5.5 & 14 & $2.8 \times 10^{-3}(0.88)$ & 250 & \multirow{3}{*}{$47(0.76)$} & \multirow{3}{*}{2.5} & \multirow{3}{*}{1.0} \\
\hline 9 & 80 & 5.5 & 14 & $7.5 \times 10^{-3}(0.98)$ & 100 & & & \\
\hline 4 & 90 & 5.5 & 14 & $6.9 \times 10^{-3}(0.92)$ & 101 & & & \\
\hline 18 & 70 & 5.5 & 20 & $9.0 \times 10^{-4}(0.98)$ & 799 & \multirow{3}{*}{76 (0.99) } & \multirow{3}{*}{2.1} & \multirow{3}{*}{2.1} \\
\hline 13 & 80 & 5.5 & 20 & $1.8 \times 10^{-3}(0.96)$ & 379 & & & \\
\hline 1 & 90 & 5.5 & 20 & $3.8 \times 10^{-3}(0.93)$ & 184 & & & \\
\hline 6 & 70 & 8 & 8 & $1.0 \times 10^{-3}(0.94)$ & 617 & \multirow{3}{*}{$68(0.95)$} & \multirow{3}{*}{1.5} & \multirow{3}{*}{2.5} \\
\hline 12 & 80 & 8 & 8 & $1.7 \times 10^{-3}(0.98)$ & 406 & & & \\
\hline 5 & 90 & 8 & 8 & $4.2 \times 10^{-3}(0.94)$ & 164 & & & \\
\hline 7 & 70 & 8 & 14 & $7.0 \times 10^{-4}(0.91)$ & 984 & \multirow{3}{*}{$115(0.78)$} & \multirow{3}{*}{8.6} & \multirow{3}{*}{1.1} \\
\hline 8 & 80 & 8 & 14 & $6.5 \times 10^{-3}(0.93)$ & 115 & & & \\
\hline 2 & 90 & 8 & 14 & $6.4 \times 10^{-3}(0.89)$ & 109 & & & \\
\hline 16 & 70 & 8 & 20 & $9.0 \times 10^{-4}(0.98)$ & 815 & \multirow{3}{*}{$81(0.99)$} & \multirow{3}{*}{1.9} & \\
\hline 17 & 80 & 8 & 20 & $2.1 \times 10^{-3}(0.98)$ & 330 & & & \\
\hline 10 & 90 & 8 & 20 & $4.1 \times 10^{-3}(0.97)$ & 170 & & & \\
\hline
\end{tabular}

En la tabla 1, también se presentan los parámetros cinéticos ( $\mathrm{t}_{1 / 2}$ y Ea) que describen la degradación térmica de FT del extracto, bajo diferentes condiciones de $\mathrm{pH}$ y SS. Allí se observa que a $14{ }^{\circ}$ Brix la mayor Ea se obtiene con $\mathrm{pH} 8$, mientras que la menor Ea y $\mathrm{t}_{1 / 2}$ se obtienen a pH 5.5, lo que indica que los FT a $14{ }^{\circ}$ Brix son más estables a pH 8, a diferencia de los resultados de Reyes y Cisneros-Zevallos (2007), quienes encontraron que los incrementos de $\mathrm{pH}$ aumentaban la velocidad de degradación de antocianinas de papa roja, papa púrpura, zanahorias y uvas, con $\mathrm{pH}$ entre 1 y 3 . Por su parte, los valores de $t_{1 / 2}$, reportados por estos autores son superiores a los del presente trabajo, posiblemente por los efectos del pH que ellos mismos observaron. Es notorio el descenso de $t_{1 / 2}$ con la temperatura para todas las condiciones evaluadas, que se debe al efecto que la temperatura tiene sobre la velocidad de degradación de compuestos fenólicos (Timberlake et al., 1980; Albarici y Pessoa, 2012; Figueirêdo et al., 2014; Zhang et al., 2014). Los valores de Ea en la mayoría de las condiciones evaluadas en el presente estudio, están por encima de los reportados por Albarici y Pessoa (2012) para bebida de açaí (49.24 kJ mol-1 a pH 5.2); jugo de mora (75.50 kJ mol-1) y pulpa de mora $(65.06 \mathrm{~kJ}$ mol-1) encontrado por Wang y Xu (2007) y pulpa de zanahoria negra $(63.7 \mathrm{~kJ}$ mol-1) reportados por Kırca et al. (2007), los cuatro últimos a pH 4.3, lo cual indica la estabilidad térmica relativa de los extractos de $B$. orellana $L$. bajo estas condiciones de trabajo. También se calculó el $Q_{10}$ en la reacción de degradación de FT en semillas de $B$. orellana L. para los intervalos de temperatura entre 70/80 y $80 / 90{ }^{\circ} \mathrm{C}$. En general estos valores presentan mayor incremento en el intervalo $70 / 80^{\circ} \mathrm{C}$ que en $80 / 90^{\circ} \mathrm{C}$, obteniéndose los mayores cambios para el intervalo $70 / 80^{\circ} \mathrm{C}$ a $14{ }^{\circ} \mathrm{Brix}$ y $\mathrm{pH} 8$, y a $8{ }^{\circ} \mathrm{Brix}$ y pH 5.5 , donde la velocidad de reacción se incrementa 8.58 y 4.10 veces, respectivamente.

Tabla 2: ANOVA para evaluar el efecto de los factores sobre la constante cinética de degradación de FT del extracto de semillas de $B$. orellana.

\begin{tabular}{|c|c|c|c|c|c|}
\hline $\begin{array}{c}\text { Fuente de } \\
\text { variación }\end{array}$ & $\begin{array}{c}\text { Suma de } \\
\text { cuadrados }\end{array}$ & $\begin{array}{c}\text { Grados de } \\
\text { libertad }\end{array}$ & $\begin{array}{c}\text { Cuadrado } \\
\text { medio }\end{array}$ & Valor $F$ & Valor - $P$ \\
\hline Modelo & $1.2 \times 10^{-4}$ & 4 & $3.1 \times 10^{-5}$ & 26 & $<0.0001$ \\
\hline $\mathrm{T}$ & $4.4 \times 10^{-5}$ & 1 & $4.4 \times 10^{-5}$ & 38 & $<0.0001$ \\
\hline $\mathrm{SS}$ & $5.6 \times 10^{-7}$ & 1 & $5.6 \times 10^{-7}$ & 0.47 & 0.50 \\
\hline $\mathrm{T}^{2}$ & $6.1 \times 10^{-6}$ & 1 & $6.1 \times 10^{-6}$ & 5.2 & 0.03 \\
\hline $\mathrm{SS}^{2}$ & $4.8 \times 10^{-5}$ & 1 & $4.8 \times 10^{-5}$ & 41 & $<0.0001$ \\
\hline $\mathrm{R}^{2}$ & 0.83 & & & & \\
\hline
\end{tabular}


Los resultados del análisis de varianza (ANOVA) para el DOE, se presentan en la tabla 2, donde se observa que $T$ y SS ejercen un efecto estadísticamente significativo $(P<0.05)$ sobre la degradación de $F T$ además, ambas variables presentan significancia en su término cuadrático. Por su parte, el pH no presentó efectos estadísticamente significativos $(P>0.05)$ sobre $k$, en el rango de valores evaluados. A partir de la ANOVA se obtiene el modelo para k (ecuación 5), que expresa la dependencia de este parámetro con los factores (T, SS), lo cual se muestra en forma gráfica en la figura 1-a. Dicha figura muestra en la parte superior los valores máximos de k para cada temperatura. Estos valores se encuentran en torno al punto intermedio del factor SS (14 ${ }^{\circ}$ Brix). Este máximo de $\mathrm{k}$ en términos de SS, se debe a la diferencia de signo entre el término lineal y el cuadrático de SS (ecuación 5), puesto que es posible que en el rango inferior de valores de SS, el término lineal (positivo) tenga preponderancia sobre el cuadrático, pero por encima de un cierto valor (alrededor de $14^{\circ}$ Brix), el término cuadrático (negativo), empieza a tener preponderancia, provocando el descenso en la repuesta.

El comportamiento en la primera parte de la gráfica (SS < 14\%) señala que el aumento de SS incrementa la degradación de FT, lo cual fue reportado por Kırca et al. (2007), quienes encontraron que los valores de k aumentaban con SS en la degradación de antocianinas de Zanahoria negra. Mientras que valores mayores de SS (por encima de 14\%) favorecen la conservación de FT, puesto que reducen los valores de k, esto puede deberse a que incrementos en la actividad de agua del medio (descensos de SS) causan degradación de las antocianinas (Garzón 2008), probablemente debido a una mayor interacción entre el agua y el catión flavilio para formar la pseudobase inestable (Olaya et al., 2009), lo anterior tiene relevancia si se tiene en cuenta que incrementos de SS provocan descensos en la actividad del agua (Zapata y Montoya, 2012). En términos de $\mathrm{T}$, la respuesta $(\mathrm{k})$ se incrementa para todo el rango de SS estudiado, a pesar de que el incremento es más marcado en los valores más bajos de $\mathrm{T}$ y después de un cierto valor el incremento es más lento, que se manifiesta con el signo diferente entre el término lineal y cuadrático de $\mathrm{T}$ en la ecuación 5 . Uno de los mecanismos por los que se pueden deteriorar los compuestos fenólicos, como es el caso de las antocianinas, se debe a que incrementos de temperatura resultan en pérdida del azúcar glicosilante en la posición 3 de la molécula y apertura de anillo con la consecuente producción de chalconas incoloras (Timberlake, 1980). Principalmente importante considerarlo a la hora de diseñar procesos de conservación térmica en sustratos que contengan FT que se quieran proteger, como es el caso de procesamiento de frutas y verduras, porque a mayor temperatura mayor tendencia a degradarse los FT, pero el efecto de SS no es igual en todos los sustratos.

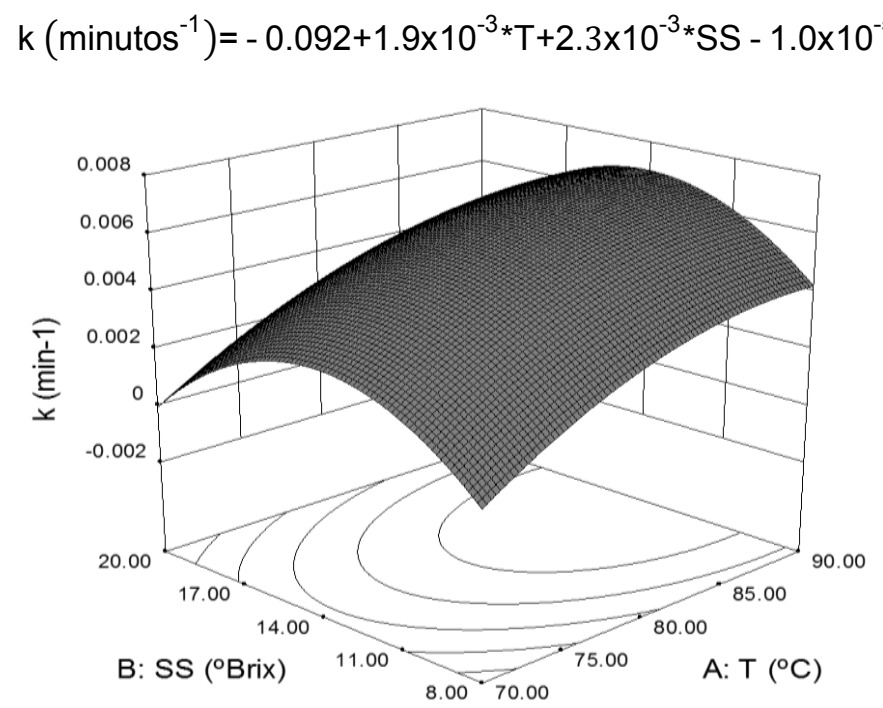

(a)

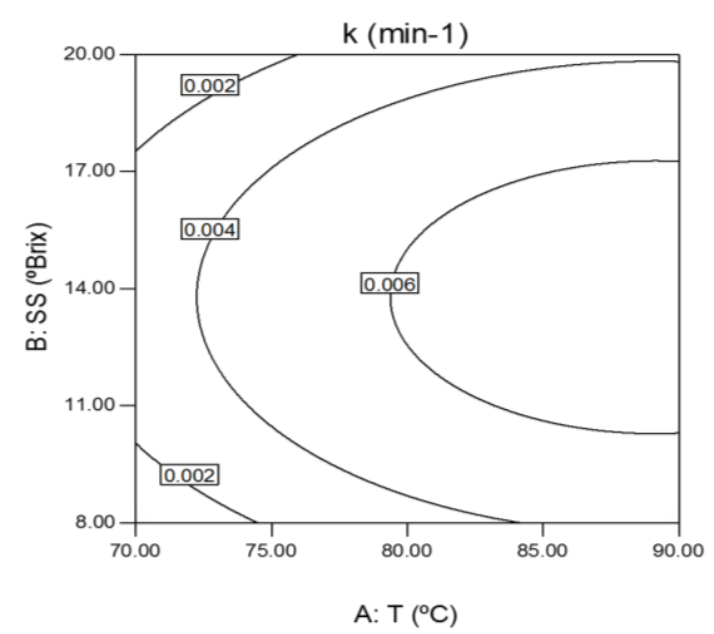

(b)

Fig. 1: Superficie de respuesta (a) y gráfico de contorno (b) para k, en función de SS y T, en la degradación térmica de FT del extracto de semillas $B$. Orellana $L$.

\section{Estabilidad de los compuestos fenólicos y la actividad antioxidante durante el almacenamiento del extracto}

En la figura 2, se presenta el descenso en la concentración de FT en extractos de semillas de $B$. orellana L., en función del tiempo a diferentes temperaturas $\left(-20,8,23\right.$ y $\left.37^{\circ} \mathrm{C}\right)$. La degradación térmica se ajustó a un modelo de reacción de primer orden, como se encontró en antocianinas de zanahoria negra (Kırca et al., 2007), de papas (Reyes y Cisneros-Zevallos, 2007) y de pulpa de açaí (Albarici, 2012). La velocidad de degradación se incrementó con el aumento de la temperatura de almacenamiento como sucedió a las condiciones de procesamiento $\left(70-90^{\circ} \mathrm{C}\right)$. 
La importancia de evaluar la estabilidad de la actividad antioxidante en función del tiempo se basa en que la es una de las actividades que más se destaca de los compuestos fenólicos (Zhang et al., 2014). En la figura 2 se presenta también el comportamiento de la actividad antioxidante medida con dos métodos diferentes, en función del tiempo para el extracto durante 91 días de almacenamiento a cuatro temperaturas diferentes (-20, 8,23 y $37^{\circ} \mathrm{C}$ ). Se observa que la actividad tiene una dependencia inversa con la temperatura, para los dos métodos. En ambos casos la pérdida de actividad siguió una cinética de primer orden como ocurrió con la degradación térmica de FT, así como en compuestos fenólicos de otras especies vegetales (Henríquez et al., 2014; Kırca et al., 2007; De Paepe et al., 2014; Fischer et al., 2013).
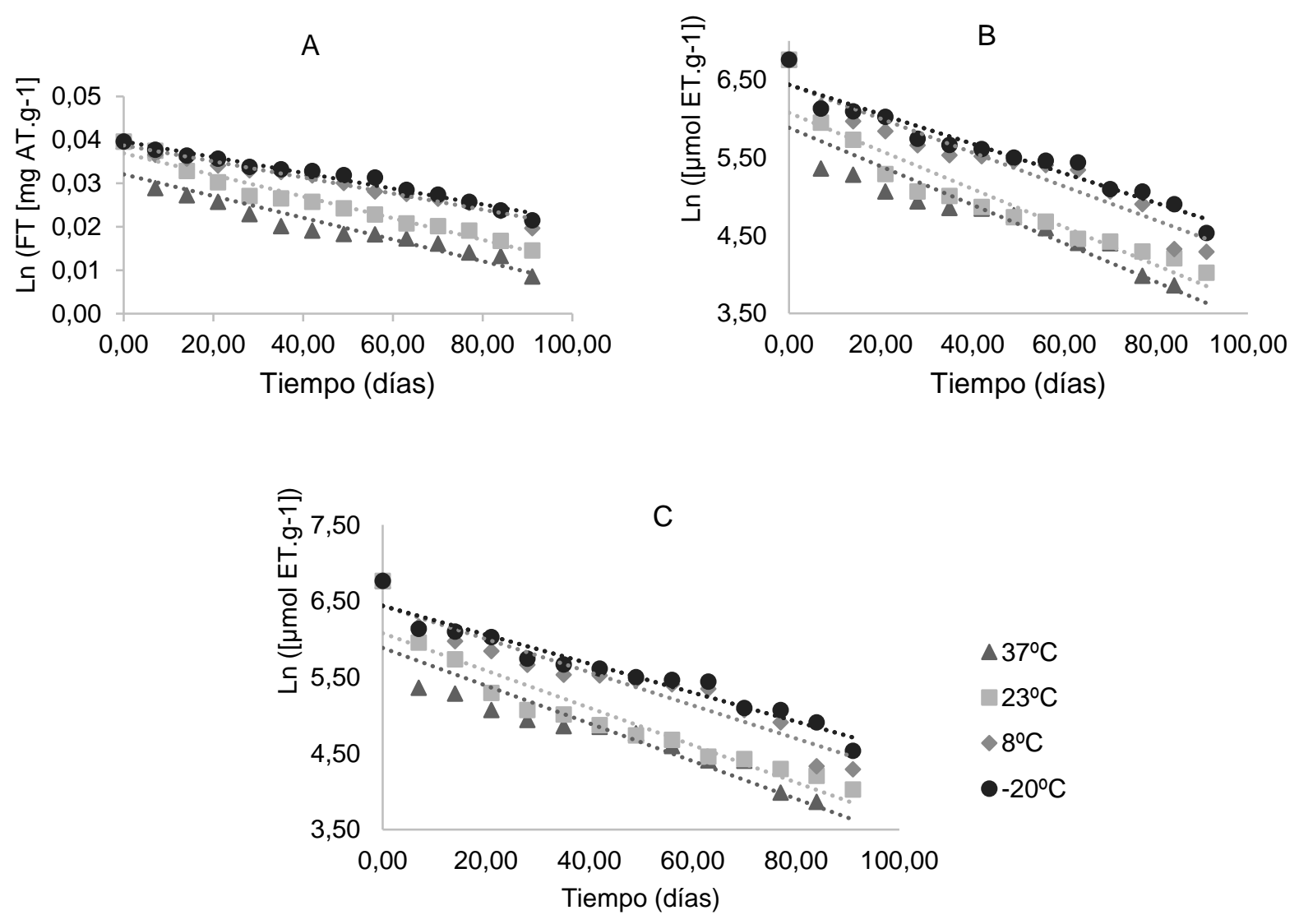

Fig. 2: Estabilidad de los compuestos fenólicos (A) y de la actividad antioxidante método ABTS $(B)$ y método FRAP $(C)$ del extracto $B$. orellana $L$. en función de la temperatura.

En la tabla 3 se presentan los parámetros cinéticos de la degradación térmica de los FT de semillas de $B$. orellana $L$., en la que se observa que la temperatura incrementa los valores de $k$ y reduce los valores de $t_{1 / 2}$, es decir, incrementa la velocidad de degradación de FT. Los efectos positivos de T sobre la velocidad de degradación de FT, ya han sido reportados en aceite de oliva, zanahoria (Kırca et al., 2007) papa roja, papa púrpura, zanahorias y uvas, (Reyes y Cisneros-Zevallos, 2007) B. orellana L., (Figueirêdo et al., 2014) pulpa de açaí (Albarici y Pessoa, 2012) y Origanum vulgare. (Zhang et al., 2014). Es importante destacar la diferencia entre los parámetros de degradación térmica que se presentan en la tabla 1, con respecto a los de la tabla 3 , debido a que las temperaturas de procesos $\left(70 / 80 / 90^{\circ} \mathrm{C}\right)$ presentan valores muy por encima de los que se tienen a las temperaturas de almacenamiento $\left(-20 / 37^{\circ} \mathrm{C}\right)$, posiblemente porque el proceso a altas temperaturas, provocan mayores deterioros de la estructura de los compuestos fenólicos, como ya se mencionó en el caso de las antocianinas, en las que incrementos de temperatura pueden resultar en apertura del anillo y pérdida del azúcar glicosilante en la posición 3 (Timberlake, 1980).

La variación en las tasas de degradación con respecto al cambio de la temperatura, expresado por el $Q_{10}$ (Tabla 3) muestra que las tasas de degradación son similares entre las temperaturas 8 y $-20^{\circ} \mathrm{C}$ y entre las temperaturas 37 y $23^{\circ} \mathrm{C}$, pero un rango de temperatura comprendido entre 8 y $23^{\circ} \mathrm{C}$, presenta un incremento mayor en la tasa de degradación. En la tabla 3 también se presentan los parámetros térmicos de la pérdida de actividad antioxidante del extracto de semillas de B. orellana L., en la que se puede observar como los valores de $k$ aumentan con la temperatura, mientras que los de $t_{1 / 2}$ se reducen, de la misma forma que se observó para los valores de la degradación de FT. 
Es de destacar que los parámetros de degradación de $\mathrm{FT}$, tienen tendencias similares y valores con un alto nivel de similitud a los parámetros de pérdida de actividad antioxidante. También se calcularon los valores de $Q_{10}$ para la degradación térmica de la actividad antioxidante del extracto de $B$. orellana $L$. a las condiciones de almacenamiento. En la tabla 3 se aprecia que el valor de este parámetro al igual que los anteriores parámetros térmicos tiene la misma tendencia y valores similares que para los compuestos fenólicos, lo cual ratifica que las propiedades antioxidantes de los extractos de $B$. orellana $L$. se deben a los compuestos fenólicos (Padmashree et al., 2014).

Tabla 3: Parámetros cinéticos de la degradación de compuestos fenólicos y actividad antioxidante durante el almacenamiento del extracto de semillas de B. Orellana L.

\begin{tabular}{|c|c|c|c|c|c|c|}
\hline \multirow{2}{*}{$\begin{array}{l}\text { Temperatura } \\
\left({ }^{\circ} \mathrm{C}\right)\end{array}$} & \multicolumn{2}{|c|}{$F T$} & \multicolumn{2}{|c|}{ ABTS } & \multicolumn{2}{|c|}{ FRAP } \\
\hline & $k\left(\right.$ Días $\left.^{-1}\right)$ & $t^{1 / 2}$ (Días) & k (Días $\left.{ }^{-1}\right)$ & $t^{1 / 2}$ (Días) & $k \quad\left(\right.$ Días $\left.^{-1}\right)$ & $t^{1 / 2}$ (Días) \\
\hline-20 & $0.018(0.97)$ & 38 & $0.019(0.84)$ & 37 & $0.019(0.67)$ & 36 \\
\hline 8 & $0.019(0.97)$ & 37 & $0.022(0.89)$ & 32 & $0.020(0.77)$ & 34 \\
\hline 23 & $0.028(0.96)$ & 28 & $0.024(0.92)$ & 28 & $0.027(0.94)$ & 26 \\
\hline 37 & $0.025(0.88)$ & 28 & $0.025(0.93)$ & 28 & $0.031(0.91)$ & 22 \\
\hline $\mathrm{Ea}\left(\mathrm{KJ} \cdot \mathrm{mol}^{-1}\right)$ & $4.1(0.74)$ & & $3.2(0.97)$ & & $5.5(0.80)$ & \\
\hline$Q_{10}(8 /-20)$ & 1.0 & & 1.1 & & 1.0 & \\
\hline $\mathrm{Q}_{10}(23 / 8)$ & 1.2 & & 1.1 & & 1.2 & \\
\hline $\mathrm{Q}_{10}(37 / 23)$ & 1.0 & & 1.0 & & 1.1 & \\
\hline
\end{tabular}

\section{CONCLUSIONES}

La degradación térmica de los compuestos fenólicos presentes en los extractos de semillas de B. orellana L., depende del aumento o disminución de T y SS, pero no del pH. La velocidad de degradación térmica de dichos compuestos sigue una cinética de primer orden, tanto en condiciones de procesamiento como en condiciones de almacenamiento. La actividad antioxidante de los extractos de semillas de B. orellana L. sufren un decrecimiento en función del tiempo y la temperatura con el mismo patrón que el de los compuestos fenólicos, por lo que se puede atribuir la actividad antioxidante de estos extractos principalmente a los compuestos fenólicos presentes en ellos. El conocimiento del efecto de las variables estudiadas sobre los compuestos fenólicos y la actividad antioxidante del extracto de $B$. orellana L., permiten mejorar el diseño de los procesos de extracción y conservación de este sustrato.

\section{NOTACIÓN}

\section{Símbolos}

$\mathrm{T}=$ Temperatura

SS= Sólidos solubles

$\mathrm{FT}=$ Fenoles totales

$\mathrm{DOE}=$ Diseño Experimental

k= Constante de reacción

$\mathrm{Ea}=$ Energía de activación

$Q_{10}=$ Coeficiente de temperatura

$\mathrm{t}_{1 / 2}=$ Tiempo medio

\section{Abreviaciones}

ABTS=2,2'-azino-bis (3-etilbenzotiazolin-6- ácido sulfónico

$\mathrm{FRAP}=$ Poder antioxidante de la reducción férrica

\section{AGRADECIMIENTOS}

Los autores agradecen a la estrategia de sostenibilidad del Comité para el Desarrollo de la Investigación en la Universidad de Antioquia (CODI), por el apoyo financiero entregado. 


\section{REFERENCIAS}

Agati, G., E. Azzarello y otros dos autores, Flavonoids as Antioxidants in Plants: Location and Functional Significance, doi: 10.1016/j.plantsci.2012.07.014, Plant Sc., 196, 67-76 (2012)

Albarici, T.R. y J.D.C. Pessoa, Effects of Heat Treatment and Storage Temperature on the Use of Açaí Drink by Nutraceutical and Beverage Industries, doi: 10.1590/S0101-20612012005000026, J. Food Sci. Technol. (Campinas), 32(1), 9-14 (2012)

Araujo J., L. Mendonça-Melo y otros tres autores, Phenolic Composition and Leishmanicidal Activity of Red Propolis and Dalbergia ecastaphyllum (L.) Taub (Fabaceae) Extracts from Sergipe, Brazil, doi: 10.1590/1678-4324-2018160461, Braz. Arch. Biol. Technol., 61, e18160461 (2018)

Bachir, M., L. Meziant y otros dos autores, Deployment of Response Surface Methodology to Optimize Recovery of Dark Fresh Fig (Ficus carica L., var. Azenjar) Total Phenolic Compounds and Antioxidant Activity, doi: 10.1016/j.foodchem.2014.04.054, Food Chem., 162(4), 277-282 (2014)

Campos R., A. Zerlotti y otros cuatro autores, In Vitro Scavenging Capacity of Annatto Seed Extracts against Reactive Oxygen and Nitrogen Species, doi: 10.1016/j.foodchem.2010.12.139, Food Chem., 127, 419-426 (2011)

Catarino M., O. Talhi y otros tres autores, Chapter 3 - The Antiinflammatory Potential of Flavonoids: Mechanistic Aspects, doi: 10.1016/B978-0-444-63602-7.00003-5, Stud. Nat. Prod. Chem., 48, 65-99 (2016)

De Paepe D., D. Valkenborg y otros siete autores, Thermal Degradation of Cloudy Apple Juice Phenolic Constituents, doi: 10.1016/j.foodchem.2014.04.005, Food Chem., 162, 176-185 (2014)

Do Prado, A.C.P., H.S. Da Silva y otros seis autores, Effect of the Extraction Process on the Phenolic Compounds Profile and the Antioxidant and Antimicrobial Activity of Extracts of Pecan Nut [Carya illinoinensis (Wangenh) C. Koch] Shel, doi: 10.1016/j.indcrop.2013.11.031., Ind Crops Prod. 52, 552-561 (2014)

Figueirêdo, B.C., I.J. Trad y otros dos autores, Effect of Annatto Powder and Sodium Erythorbate on Lipid Oxidation in Pork Loin During Frozen Storage, doi: 10.1016/j.foodres.2014.07.016, Food Res. Int. 65(2), 137-143 (2014)

Fischer, U.A., R. Carle y D.R. Kammerer, Thermal Stability of Anthocyanins and Colourless Phenolics in Pomegranate (Punica granatum L.) Juices and Model Solutions, doi: 10.1016/j.foodchem.2012.10.072., Food Chem. 138(2-3), 18001809 (2013)

Gad S., E. Motaal y otros tres autores, Cytotoxic activity of phenolic constituents from Echinochloa crus-galli against four human cancer cell lines, Rev. bras. Farmacogn. 26, 62-67 (2016)

Garzón, G.A., Las Antocianinas como Colorantes Naturales y Compuestos Bioactivos: Revisión, Acta Biol. Colomb. 13(3), 27-36 (2008)

He, X.L., X.L. Li y otros dos autores, Composition and Color Stability of Anthocyanin-Based Extract from Purple Sweet Potato, doi: 10.1590/1678-457X.6687, J. Food Sci. Technol. (Campinas), 35(3), 468-473 (2015)

Henríquez, C., A. Córdova y otros dos autores, Kinetic Modeling of Phenolic Compound Degradation During Drum-Drying of Apple Peel By-Products, doi: 10.1016/j.jfoodeng.2014.06.037, J. of Food Eng. 143, 146-153 (2014)

Kırca, A., M. Özkan y B. Cemeroğlu, Effects of Temperature, Solid Content and Ph on the Stability of Black Carrot Anthocyanins, doi: 10.1016/j.foodchem.2006.01.019, Food Chem., 101(1), 212-218 (2007)

Koolen, H.H.F., F.M.A. Da Silva y otros tres autores, Antioxidant, Antimicrobial Activities and Characterization of Phenolic Compounds from Buriti (Mauritia flexuosa L. f.) by UPLC-ESI-MS/MS, doi: 10.1016/j.foodres.2013.01.039, Food Res. Int. 51(2), 467-473 (2013)

Lima, N., L. Cursino-Hron y otros cinco autores, Antifungal activity of extracts and phenolic compounds from Deguelia duckeana, doi: 10.1016/J.BJP.2018.08.004, Rev. Bras. Farmacogn., 28(6), 697-702 (2018)

Montgomery, D.C., Diseño y Análisis de Experimentos, 5ª Ed., Limusa Wiley, México D.F., México (2005)

Moo-Huchin, V.M., M.I. Moo-Huchin y otros seis autores, Antioxidant Compounds, Antioxidant Activity and Phenolic Content in Peel from Three Tropical Fruits from Yucatan, Mexico, doi: 10.1016/j.foodchem.2014.05.127, Food Chem. 166, 17-22 (2015)

Olaya, C., M. Castaño y G. Garzón, Stability of Anthocyanins from Rubus Glaucus and Solanum Betaceum Cav.Dark-Red Strain as Affected by Temperature, Storage and Water Activity, Acta Biol. Colomb., 14(3), 143-158 (2009)

Padmashree, A., G. Sharma y otros dos autores, Antioxygenic Activity of Solanum nigrum L. Leaves in Sunflower Oil Model System and Its Thermal Stability, doi: 10.4236/fns.2014.511113, Food Nutr. Sci. 5(11), 1022-1029 (2014)

Pulido, R., L. Bravo y F. Saura, Antioxidant Activity of Dietary Polyphenols as Determined by a Modified Ferric Reducing/Antioxidant Power Assay, doi: 10.1021/jf9913458, J. Agric. Food Chem., 48(8), 3396-3402 (2000)

Re, R., N. Pellegrini y otros cuatro autores, Antioxidant Activity Applying an Improved ABTS Radical Cation Decolorization Assay, doi: 10.1016/S0891-5849(98)00315-3, Free Radic. Biol. Med. 26(9-10), 1231-1237 (1999)

Reyes, L.F. y L. Cisneros-Zevallos, Degradation Kinetics and Colour of Anthocyanins in Aqueous Extracts of Purple- and Red-Flesh Potatoes (Solanum tuberosum L.), doi: 10.1016/j.foodchem.2005.11.002, Food Chem., 100(3), 885-894 (2007) 
Silva, V., G. Igrejas y y otros siete autores, Chemical composition, antioxidant and antimicrobial activity of phenolic compounds extracted from wine industry by-products, doi: 10.1016/j.foodcont.2018.05.031, Food Control, 92,516-522 (2018)

Singleton, V.L. y J. Rossi, Colorimetry of Total Phenolics with Phosphomolybdic-Phosphotungstic Acid Reagents, Am. J. Enology Vitic. 16(3), 144-158 (1965)

Timberlake, C.F. Anthocyanins Occurrence, Extraction and Chemistry, doi: 10.1016/0308-8146(80)90065-5, Food Chem., 05 (1), 69-80 (1980)

Wang, W.D. y S.Y Xu, Degradation Kinetics of Anthocyanins in Blackberry Juice and Concentrate, doi: 10.1016/j.jfoodeng.2007.01.018, J. of Food Eng., 82(3), 271-275 (2007)

Zapata, J.E. y A. Montoya, Deshidratación Osmótica de Láminas de Mango cv. Tommy Atkins Aplicando Metodología de Superficies de Respuesta, Rev. Fac. Nac. Agron. Medellín, 65(1), 6507-6518 (2012)

Zhang, X.L., Y. S. Guo y otros siete autores, Phenolic Compounds from Origanum Vulgare and Their Antioxidant and Antiviral Activities, doi: 10.1016/j.foodchem.2013.11.153, Food Chem., 152, 300-306 (2014) 
